\title{
Research on train anti-collision method based on deep learning
}

\author{
Jianming Zhang ${ }^{1, a}$, Qijin Lü,b, ShutingZaoo,c \\ ${ }^{1}$ Traffic Control Technology CO, Ltd, Beijing, China \\ ${ }^{2}$ Traffic Control Technology CO, Ltd, Beijing, China \\ ${ }^{3}$ Traffic Control Technology CO, Ltd, Beijing, China
}

jianming.zhang@bj-tct.com, qijin.lu@bj-tct.com, shuting.zao@bj-tct.com

\section{Key Words:Ranging, Collision Avoidance, Safe Driving, Driving Efficiency}

\begin{abstract}
Real-time detection of train distance from the front of the obstacle to ensure that the train in the case of safe braking speed driving is to improve the efficiency of train driving the main way. In this paper, a collision avoidance method based on deep learning is proposed. The system acquires the obstacle, the orbit environment and the signal status in front of the train through the long and short focus cameras, and then uses the depth learning image processing method to give the distance between the vehicle and the obstacle and the signal color. On the basis of ensuring safe braking, a recommended speed and driving route are given to the user, and when the signal light is in a red state and the current train is far away from an obstacle, voice prompts and alarms with different frequencies are respectively provided to ensure the safe and effective running of the train, Multiple test data show that this method can accurately determine the obstacle distance and orbit environment and signal status, and can give different voice prompts according to demand, to ensure the safe driving of the train and improve the driving efficiency and train defense Hit the coefficient, with good practicality.
\end{abstract}

\section{Introduction}

To meet the needs after the fault of the signal system, this anti-collisionsystem uses deep learning technology to make a train operation control schemein case ofthe emergency of signal system fault.The final system meets the requirements of train operation efficiency under the condition of signal system failure and emergency,and provide a back-up way for further operations, and strongly support the safe and efficient operation of subway.After the test on the actual subway lane by using generic technology, this system can support fully automatic train systems and the next generation system with independent control of trains and show the improvement of technology. 


\section{The present situation of research and development at home and abroad}

Through the investigation of the application and research of technology in the field of transportation at home and abroad, identify the advantages and disadvantages of various ways tomake a technical scheme for this project.

At present, the main ways to handle the train operation in the dispatching center are as follows:

The traditional signal system achieves the train position by measuringand integrating speed by speed measuring motor, radar and other sensors.Position correction is achievedby transponder interval. Use track circuit assistant or axle counting to get a rough positioning of a zone ${ }^{[1]}$. Upload those through a dedicated network such as WLAN or LTE to Center Station Map and show.

Tramcar system usually uses satellite positioning due to it runs mostly in open space.

It uploads to the center through the public network or private network to display ${ }^{[2]}$. The requirement of positioning accuracy is not high.

In the aspect of train collision avoidance control, there are mainly the following process methods:

Signal system master the whole train situation through ground equipment, including speed, position, etc., and carry out move authorization calculation, and control the distance and speed of trains.

Self-driving cars realize recognition of vehicle, lane, pedestrians and signal lights through image recognition by deep learning and LIDAR detection technology.Then calculates car speed and direction according to distance between obstacle and lane recognition, and controls vehicle.

This system is a backup application in the case of signal system failure. According to the technical level at home and abroad and the present situation in the industry, we absorb the advantages of various technology, and make an integrated scheme for the application of this scenario, and make a safe and reliable, simple system structure, easy to install and maintain, low cost spare system.

\section{The main content of the design}

\section{System structure design}

This system uses the image recognition algorithm based on deep learning to protect the train running environment. Alarm according to the preset tracking distance. To realize the intelligent train 
control, and to strengthen the level of the system safety and protection.

\section{Image acquisition and processing}

Image recognition model based on image information, analysis and extraction of image features, and then establish classifier. Image recognition is a technology ${ }^{[3]}$ to classify and recognize according to the characteristics of image.

In this study, we first establish classifiers for track, train, turnout and signal, then capture video frames by long and short focal cameras, and then each frame captured is processed as follows:

(1) grayscale

(2) edge detection

(3) threshold segmentation

(4) removal of noise points in rough edges

(5) edge center extraction

(6) feature extraction

At last, compare the feature values in this image with the classifier values, and identify the current devices with this feature are orbits or other devices.

\section{Distance measurement and recognition}

(1) Distance measurement

In this study, the distance measurement is mainly used to measure the distance between the end of the track and the current position of the long and short focus camera, and the distance between the train position and the current position in the long and short focus camera.

In the course of distance measurement in this study, the first need to be calibrated.

The values of the track and the distance between the position of the train and the current position are measured by a laser rangefinder (In this study, the value of the laser rangefinder is accurate ${ }^{[4]}$.The value is then written to the current system, which is calibrated repeatedly.In the future range finding process, we only need to detect the track in the image or the pixel rows of the train in the image and calculate the distance between the current train or track through the 
calibration value $^{[5]}$.

\section{(2) Recognition}

In this study, recognition is mainly about identifying the color of the signal. The model of the classifier is used to determine the gray level and the color of the signal light.

In this study, the accuracy of target recognition is determined by the matching degree of training samples in the classifier. A matching model is finally formed through a large number of training samples, and the effect of image recognition is as follows:
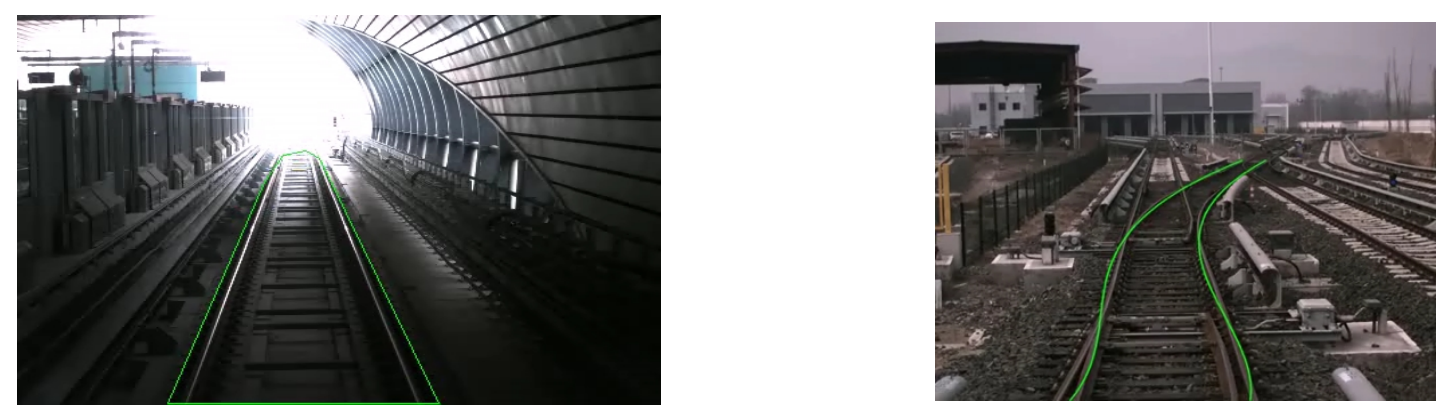

\section{system function}

\section{Auxiliary anti-collision of the train}

This system uses image recognition to monitor whether there is a train in front of the road and returnthe distancebetweenthem. Then according to the maximum braking distance gives a recommended speed. When there is a signal light beside the road ahead, such as arriving or departing from a station, meeting a switch etc., this system will identify the color of the current signal light by the image recognition technology. If it is a red light, the train shouldstop, this system will alert the driver thatin front of the road has a red light.

\section{Conclusion}

\section{Technological innovation point}

The technical innovations of this system are as follows:

1. Recognition technology based on deep learning

Deep learning is a neural network that mimics the human brain for analysis and learning. Through deep learning, it can avoid the tedious and unilateral features of artificial selection. According to the 
characteristics of the fixed line of rail transit, using image and point cloud data to optimize the recognition effect through machine training, learn and identify by machines automatic selection. [6][7]

\section{Application scene}

The application scene of the system in the train control system is as follows:

1. Whenthere is overall failure of signal system, signal system transfers to and use inter station block ${ }^{[8]}$ : under the condition that the number of trains in the zone is difficult to be judged, the driver will be aided by the early warning of the front train and red light to improve driving safety.

2. When single or multiple train vehicle signal equipment is failure: the driver of the train will be aided by the early warning of the front train and red light to improve driving safety.

3. When communication train rescues a fault train: the driver of the communication trainwill be aided by the early warning of the fault train distance ahead. ${ }^{[9]}$

4. Assist maintenance vehicle: placed on a maintenance vehicle that is not equipped with a signal system, the driver of the train will be aided by anti-collision warning.

5. Reduce supervisors: assist drivers by early warning to reduce the number of supervisor on the trainwho remove ATP.

In general, this system can save manpower cost, improve operation efficiency and improve operation safety. After the system is tested by two car tests, the scope of the test can be further extended, to test its reliability. After successful tests, the system can be divided into stages and popularized in the whole road network.

\section{Acknowledgements}

This work is supported by the Beijing major science and technology project(Project Number:Z161100005016079).

\section{References}

[1] Qing Xia.Application of transponder and velocity measurement combination in Subway [J] Railway Signalling\& Communication Engineering, 2012 , 09(2):50-53.

[2]LI Lin;ZHANGLi-ping;LI Song. Methods of nearest neighbor guery in road network with barriers. Railway Computer Application, 2009, 18(11): 37-40. 
[3]Luo S D, Ying H U. Image identification and classification model based on sample analysis[J]. Application Research of Computers, 2007, 24(11):309-311.

[4]Xinyao Zhuang.Research on the application of laser ranging in camera calibration[D].Changchun University of Technology, 2013.

[5]Jin-ling DU, Chang-hong GOU. Design and implementation of Railway Wagon Out-of-gauge Measurement System based on industrialcontrol computer[J]. Railway Computer Application, 2012, 21(9): 38-40.

[6] Guanglei Zhang.Study on deep learning based on immune convolution neural network[D].Donghua University, 2016.

[7]Xiaohu Han,Peng Xu,Sensen Han.Theoretical overview of deep learning[J].Computer Era, 2016(6):107-110.

[8] Shi Li. Application of automatic inter station block for axle counting[J]. Railway Signaling\& Communication, 2007, 43(7):16-18.

[9] Hao Liu.Research on Driver Assistance System for Train[D].Beijing Jiaotong University,2014. 